Insight, part of a Special Feature on Assessing Risks to Wildlife

\title{
Tackling Biocomplexity with Meta-models for Species Risk Assessment
}

\author{
$\underline{\text { Philip J. Nyhus }}^{1}, \underline{\text { Robert Lacy }}^{2}, \underline{\text { Frances R. Westley }}^{3}, \underline{\text { Philip Miller }}^{4}, \underline{\text { Harrie Vredenburg }}^{5}, \underline{\text { Paul Paquet }}^{6}$, and \\ $\underline{\text { John Pollak }}^{7}$
}

\begin{abstract}
We describe results of a multi-year effort to strengthen consideration of the human dimension into endangered species risk assessments and to strengthen research capacity to understand biodiversity risk assessment in the context of coupled human-natural systems. A core group of social and biological scientists have worked with a network of more than 50 individuals from four countries to develop a conceptual framework illustrating how human-mediated processes influence biological systems and to develop tools to gather, translate, and incorporate these data into existing simulation models. A central theme of our research focused on (1) the difficulties often encountered in identifying and securing diverse bodies of expertise and information that is necessary to adequately address complex species conservation issues; and (2) the development of quantitative simulation modeling tools that could explicitly link these datasets as a way to gain deeper insight into these issues. To address these important challenges, we promote a "meta-modeling" approach where computational links are constructed between discipline-specific models already in existence. In this approach, each model can function as a powerful stand-alone program, but interaction between applications is achieved by passing data structures describing the state of the system between programs. As one example of this concept, an integrated meta-model of wildlife disease and population biology is described. A goal of this effort is to improve science-based capabilities for decision making by scientists, natural resource managers, and policy makers addressing environmental problems in general, and focusing on biodiversity risk assessment in particular.
\end{abstract}

Key Words: biocomplexity; endangered species; human dimension; meta-model; population viability analysis; risk assessment; VORTEX.

\section{INTRODUCTION}

Recent advances in the science of conservation, from geographic information systems to conservation genetics, have enhanced our ability to conserve species and ecological communities. However, our ability to understand the complex factors that ultimately result in declines, and the extinction of wildlife populations is more limited (Lacy and Miller 2002). Although we know that certain threats, such as overharvesting and land cover change, can be inimical to the survival of many wildlife populations, we have more limited understanding of the interactions among complex biological, physical, and human phenomena that create these situations (Kates et al. 2001, Folke et al. 2002, Swart et al. 2002). This is particularly true when considering the challenge of trying to predict the impact of multiple biotic and abiotic stressors based on evidence from single-stressor studies (Vinebrooke 2004).

In recognition of the need to address these and other complex environmental problems, the National Science Foundation (NSF) initiated in 1999 the "Biocomplexty in the Environment" program to provide a mechanism to fund research that is by definition cross-cutting, exploring properties emerging from the interactions among behavioral, biological, chemical, physical, and social systems at multiple scales (Colwell 2001, Michener et al. 2001). At its core, this biocomplexity approach is intended to breach temporal, conceptual, and spatial boundaries, and encourage better understanding of systems that may exhibit emergent or unexpected properties that cannot be easily predicted by 
studying the behavior of individual components of the system in isolation (Michener et al. 2001). One outstanding question is whether this "biocomplexity approach" really has utility for solving the important environmental questions it was designed in part to better understand. Is this program just new jargon for interdisciplinary research or does it provide new solutions for solving environmental problems? In this paper we use our own experiences to ask whether a biocomplexity approach can help to better understand the current biodiversity crisis in general and risk assessment of endangered species in particular, and explore the constraints to implementing this approach to solve biodiversity conservation problems. We suggest one theoretical and conceptual approach to framing biodiversity risk assessments to systematically address what is an overwhelmingly complex problem.

\section{THE BIOCOMPLEXITY NETWORK}

For the past seven years, a group of us informally known as the Biocomplexity Network has been working together to create modeling, process, evaluation, and teaching tools for biodiversity risk assessment. The evolution of the Network was a response to the lack of integrated study and understanding of issues of complexity that were preventing progress on risk assessments, such as how to incorporate the very different models and diversity of topics and approaches used by social scientists in a field historically dominated by biologists. We recognized that predicting risk to endangered species requires more than specialized modeling expertise and biological knowledge, and we pursued collaboration between natural and social scientists (Nyhus et al. 2002). We also realized that understanding how such interdisciplinary networks function could be as important as the models, predictions, and policy suggestions they developed (Westley and Vredenburg 2003).

In our earliest efforts, we tried to better understand how interdisciplinary groups function, how to include more diverse groups in dialogue, how to identify constraints to their involvement in risk assessment processes, and how to apply these lessons in real case studies (Westley and Miller 2003). One of the most challenging issues we addressed through this research experience was to determine the means by which we could most effectively engage all important stakeholder domains at an early stage in the organization of species or population-level risk assessment deliberative processes. Such engagement is critical to the success of collaborative biodiversity conservation, but this is also the piece of intellectual real estate that is the most unfamiliar to the traditionally trained conservation biologist. Our research led us to the conclusion that proper problem definition is key to enticing a diversity of interests to participate in collaborative conservation: to gain the support of a particular constituency in the context of successful management of biodiversity, the issues surrounding conservation of a given species or population must be framed in a way that is relevant to the continued viability of the constituency itself (Westley et al. 2003). Shared interests will build collaboration, which can be nurtured and strengthened into longer-term partnerships to the benefit of multiple interests.

In addition to these "process learnings," we also focused on expanding existing simulation models to allow for more informative inclusion of social science data in endangered species risk assessments. This research quickly exposed the limited capabilities of traditional models of wildlife demography or the newer population viability analysis (PVA) models (Reed at al. 2002) to include the driving forces arising from human activities behind threats to biodiversity (Caughley 1994, Lacy and Miller 2002, Miller and Lacy 2003). The outputs of models of human activities and demography were not specific enough to be translated into the estimates of habitat fragmentation, degradation, and loss that were required by the existing models of wildlife population processes. We found that our own use of PVA followed a pattern that is perhaps too common. Wildlife population biologists were left to make simplistic attempts to guess at the extents to which human activities, about which we lacked expertise, would affect specific parameters of population models. We sought to broaden risk assessment processes to include such stakeholders as landowners, industry representatives, and indigenous peoples. This too proved difficult. As stated earlier, social scientists were often alienated by natural scientists problem and domain definition. Social scientists studying business organizations, for example, focus their investigations on how business leaders in resource extraction industries make strategic decisions in increasingly complex business environments framed by public concern over biodiversity decline and global climate change (Hall and Vredenburg 2003). Industry and local stakeholders, on the other hand, were alienated by 
an emphasis on seemingly abstract data gathering and modeling at the expense of moving toward local landscape-based solutions (Vredenburg 2003).

In response to these problems, we stepped back from the precise and narrow models applied within the fields of population and conservation biology to explore large-scale heuristic models as a way to broaden our thinking about what questions, data, and solutions can emerge when biological systems are seen as interdependent with forces outside of population biology. This helped us identify what expertise was necessary to include in a risk assessment workshop process as a means of building collaborative potential among diverse stakeholder groups. With this heuristic device at our disposal, we then struggled with how to develop a comprehensive simulation-modeling environment that would include the intertwined processes from many disciplines. However, we quickly grew skeptical that such "mega-models" would be broadly useful in biodiversity risk assessments. Attributing results to specific processes or data within the models is difficult at best. Also, construction of fully integrated models under this approach frequently must start from scratch rather than as an addition to well-tested models already in use, although a growing number of successful examples exist that use separate components, such as the IMAGE Development Group at the Dutch National Institute of Public Health and Environmental Protection http://www.ciesin.columbia. edu/datasets/rivm/image2.0-home.html. With the mega-model approach proving too unwieldy to be used in practice, the Biocomplexity Network began to conceptualize an alternative approach to integrating data and expertise across diverse disciplines. But successful integration meant overcoming equally diverse constraints.

\section{CONSTRAINTS TO COUPLING HUMAN- NATURAL SYSTEMS}

The environment consists of biological, physical, and human domains. Traditionally, academic disciplines focus on just one of these, or perhaps even a small subset of one, although some fields such as ecology often look at interactions between two and a few fields like geography have a history of exploring all three domains. A number of attempts have been made to develop common units of measurement that can cross these boundaries; notably H. T. Odum and colleagues developed the concept of embodied energy, or "emergy," (Odum 1996, Odum and Odum 2000, Brown and Ulgiati 2004). In general, however, growing specialization within disciplines has meant that analytical, computational, and social tools for exploring the dynamics between the biological, physical, and human domains are not nearly as well developed as are tools for addressing within-domain problems. Each domain can be further partitioned among scales of space, time, degree of reductionism, and quantification. Disciplines within each domain tend to focus on processes at one end of these scales, and these differences in scales of analysis further hinder study of the complexity that lies at the intersection of domains and across scales. Thus, although the idea of incorporating different disciplinary data and perspectives is conceptually straightforward, the roadmap to achieving that goal is constrained by both social and institutional challenges related to interdisciplinary communication, as well as limitations of typical computer modeling approaches.

\section{Social and institutional constraints}

Our understanding of how to develop research collaborations that effectively integrate the expertise of fields that span the breadth of biological, physical, and human domains is quite limited, although the topic has attracted valuable scholarly attention (Somerville and Rapport 2000, Wondolleck and Yaffee 2000). Just as it is difficult to pass data from different scales and domains among disciplinary models, it is difficult to bridge disciplinary and communication barriers among experts (Nicolson et al. 2002). Strategies for accomplishing this at broad levels have been proposed (Heberlein 1988, Clark and Wallace 1998, Machlis 1998, Wilson 1998, Pickett et al. 1999, Redman 1999, Wear 1999, Lacy and Miller 2002), but hurdles to carrying out interdisciplinary discourse remain and social scientists typically have a very limited role in the development of approaches to predict extinction risks for threatened species (Lindenmayer et al. 1993, Lacy and Miller 2002, Nyhus et al. 2002).

\section{Modeling constraints}

Effective development of models to predict species vulnerabilities is constrained by several challenges. First, the drivers of threats such as growing human 
populations or the introduction of exotic species vary dramatically along spatial and temporal scales and across disciplinary domains (Levin 2000). Biological populations may be measured at mesoscales of tens of kilometers and their extinction risk measured in decades, viruses causing disease epidemics may operate at microscales of hours or days, and atmospheric change may operate at global macroscales across many centuries (Holling et al. 2002). The interactions of flows among physical, biological, and human domains are not as well understood as the dynamics within subcomponents of each domain (Holling and Gunderson 2002). Our understanding of coupled human-natural systems is further confounded by non-linear interactions, heterogeneity of components, and flows among component parts (Levin 1999). Recent efforts to understand complex systems highlight the need to incorporate such complexities into predictive models (Gunderson and Holling 2002, Nicolson et al. 2002).

Second, few existing models are robust enough to incorporate diverse inputs from multiple domains and multiple spatial and temporal scales. One approach to this problem has been to develop megamodels from the ground-up (Vanclay 1998). These highly sophisticated models are typically developed by teams of researchers to address complex but focused research problems. These models benefit from the cohesiveness that comes from development by a single team, up-front understanding of parameters and limits, and development within a standardized modeling environment. A major drawback to such models is their potential inflexibility because they are designed to address a predefined problem and their size and complexity restrict their utility to the original developers.

Another approach has been to tackle just one stratum of a complex problem. For example, success has been achieved through the use of global circulation models for understanding the effect of increasing carbon dioxide on atmospheric systems (Watson 2001). Such models are well suited to understand physical atmospheric changes over time or even broad impacts on ecosystems, but are less well suited to address problems at much finer scales across different domains, such as how animals in a wildlife population will respond to changing temperatures (Gitay et al. 2002).

Yet another approach has been to break down complex problems into scales and levels of complexity that are more manageable. Efforts to date to define species extinction risks have typically succeeded best where systems are narrowly bounded and data are widely available. For example, population viability analysis (PVA) models based on population biology are used widely to project fates of wildlife populations under alternative scenarios reflecting future management regimes (Boyce 1992, Burgman et al. 1993, Lindenmayer et al. 1993, Sjögren-Gulve and Ebenhard 2000, Beissinger and McCullough 2002, Morris et al. 2002, Reed et al. 2002). PVA models have been shown to be able to project population trends and vulnerability for simple systems with well delimited populations in relatively unchanging environments (Brook et al. 2000, Lindenmayer et al. 2000), but accuracy may be limited over longer time frames and when input data are imprecise (Ellner et al. 2002), and simple PVA models may be less robust for predicting fates of metapopulations in changing landscapes (e.g., Lindenmayer and Lacy 2002; Lindenmayer et al. 2003).

One important criticism of PVA models is that many focused on demographic rates in the wildlife populations but did not consider as effectively the many other biological, physical, and social processes that may be driving changes in these rates. Simple models of wildlife demography do not capture the complexity of interactions among biological, physical, and human systems and their variability over time and space, although there has been some work to integrate PVA analyses with factors such as timber harvest (Lindenmayer and Possingham 1995, Lindenmayer and Possingham 1996), and ongoing land conversion, for example with the Spotted Owl (Lamberson et al. 1992), Redcockaded Woodpecker (Plentovich et al. 1998), and Bachman's Sparrow (Liu et al. 1995). However, other wildlife population models have been combined with alternative futures in land use policy using programs like PATCH (e.g., Schumaker et al. 2004).

\section{TOWARD AN OPEN-DATA APPROACH TO DEVELOPING META-MODELS}

Given the dual challenges of (1) identifying the information and expertise from diverse disciplines that is required to approach a complex species conservation issues, and (2) trying to develop insightful quantitative models that can successfully bridge the data domains across these disciplines, 
there is a need to develop tools that address both problems.

To link and integrate knowledge across disciplines, we have begun building "open-data meta-models" to provide one approach to allow information to flow among computer models developed within specific disciplines. In an open-data meta-model, the specifications of the input parameters for each component submodel and its descriptors of the system are stored on the computer system in a form that is accessible to other programs. Each program has access to the data structures of each other program, using and possibly changing the values generated by other programs. To allow dynamic interaction among models, each model provides function calls within a dynamic link library (dll) for initialization, incrementing time steps, and closing. In this way, any one model, or a higher-level interface program, can provide the interface through which users view the overall system. To capture the advantages of open-data models requires that data specifications be widely available, e.g., via the Internet, so that potential users and contributors have access.

This meta-modeling approach is both more complete and more efficient than what would likely emerge from an attempt to create a singular model of a system. The strengths of existing models are retained, rather than subsystems being simplified to force them into a de novo representation developed by practitioners from other fields. Coding effort and errors are minimized, as algorithms to represent complex components are not reinvented and reimplemented. The components driving the dynamics of the overall system can be more easily discerned because subsystems are easily separable or combinable, rather than being inextricably intertwined within the model. The conceptual advantages of meta-models for understanding complex systems are parallel to the advantages to coding, debugging, and software maintenance that arise from object-oriented programming.

Meta-models will not solve all of the limitations inherent in using models to understand the complex processes that impact species viability. Any model that invokes chains of events can suffer badly from error propagation that makes long-term predictions unreliable (Ellner et al. 2002). A meta-model does allow analysis of the contributions of uncertainty in each component, but only if practitioners will compare the projections from independent models to those resulting from the linked meta-model. Moreover, the use of a meta-model to include several component systems in an analysis does not guarantee that the relevant component processes have been considered and, as with most modeling approaches, there will be a tendency to use the tools, e.g., linkable submodels, that are readily available rather than assessing more openly what are the likely drivers of the overall system. For this reason, we are working to develop a meta-modeling framework that can make links among any models that follow some simple rules for data transfer.

Meta-models can improve upon the more traditional reliance on separate analyses that implicitly assume independence of processes and therefore that the overall complex biophysical-social system can be assessed simply as the sum of its parts. By even loosely coupling component models through passing data back and forth while each is running, meta-models will allow for nonlinear feedback and emergence of unexpected trends. However, if the detailed processes within each component subsystem are truly intertwined intimately with the processes within others, then a fully integrated mega-model may be necessary to represent adequately that complexity. We envision metamodels as a flexible way to work in between the extremes of independent analyses from divergent disciplines and a fully holistic approach that demands development of a comprehensive model that integrates knowledge systems into one.

Although we are just now developing highly flexible meta-model tools, we hypothesize that open-data meta-models will promote the study of interactions by making the components modeled by each discipline available for use by others. As an example of this, inclusion of a genetic submodel in the VORTEX PVA software, a simulation for projecting the dynamics of wildlife populations (Lacy 1993, 2000), has promoted consideration of the interactions between genetic and demographic threats to population viability (Lacy and Lindenmayer 1995), and has led to experimentation to test patterns predicted from the models (Lindenmayer et al. 1999). We have also linked VORTEX to a model of genetic management of populations to project the consequences of management strategies. We are currently developing an epidemiological modeling program (OUTBREAK) for simulating disease in wildlife populations, creating a two-component meta-model by linking it to VORTEX (Fig. 1). In the linked meta- 
model, a VORTEX user calls on OUTBREAK to simulate the dynamics of disease within each year of the simulation. Working in the alternative interface, an OUTBREAK user can ask VORTEX to provide the population changes. The data describing the state of the system can be used or changed from either side. For example, susceptibility to disease, specified within OUTBREAK, can be a function of the genes of individuals or population density, each modeled within VORTEX, whereas mortality or reproduction, specified within VORTEX, can be functions of disease state, modeled within OUTBREAK. More recently, we have developed a prototype agent-based model of animal dispersal that incorporates spatial models of landscape change from a geographic information system. The model of animal dispersal can then be linked to VORTEX, passing to it survival and other demographic rates applicable to the location of each animal.

At Riding Mountain National Park, Canada, we explored the feasibility of creating a three-legged meta-model with collaborators to explore the dynamics of tuberculosis transmission between elk and livestock using these three linked models. By explicitly linking these models, we can explore questions that otherwise would have been much more challenging. For example, how will killing elk influence both the viability of the resident elk population and the probability of tuberculosis transmission to surrounding livestock? How will selectively culling in some areas, or altering the permeability of barriers, e.g., fences, influence these processes? Individually, the component models can answer parts of these questions. But when combined, these meta-models offer an entirely new level of feedback and analysis while leaving the original models and disciplinary focus intact.

Interactions between demographic and genetic processes with disease are just one example of models that can be linked as in some epidemiological research (Haydon et al. 2002). However, even this example is still within the comfortable realm of biocomplexity as it involves processes restricted to the biological domain. Our meta-model approach could be used to further examine virtually any problem in which the relationships between subcomponents of the system can be linked. Building on our earlier heuristic model, possible next steps could include directly linking models of harvest, human-wildlife conflict, or other social or biophysical models into this analytical platform. Using this approach, virtually any model in which we can specify the data to be shared can be linked to our PVA approach. We further hypothesize that these linkages will lead to understanding within fields that was not apparent before and that meta-models will better reveal emergent properties of the interactions between systems. As noted above, in parallel with explorations of our biocomplexity network, there has been exciting growth in efforts to incorporate the human dimension into biodiversity risk assessments. Many of these incorporate a casespecific mega-model approach, which will likely preclude widespread use for many endangered species threat assessments and may not facilitate the collaborations among disciplinary experts that we feel is needed to achieve conservation success. Although work on all levels of complexity are needed, we hope the concept of linking independent models of disease or harvest or conflict or forest change to existing PVA models, whether VORTEX, $P A T C H$, or other models, will provide an accessible approach that allows specialists to engage in conservation assessments by focusing on their own areas of expertise. This should allow the best considered models within each discipline to be used, with those experts providing the underlying data, understanding of possible sources of error, and interpretations of outcomes that would then be synthesized into more complete understanding arising from linkages among models and among people.

\section{Beyond quantification: scenario testing}

One challenge of working with multiple stressors is the diversity of scales and divergence of data that make quantitative predictions difficult. Scenario testing is increasingly being used to integrate knowledge of divergent types to encapsulate the understanding at one higher, more qualitative, holistic, or causal level in a way that defines the processes that need to be represented and examined in the lower more mechanistic models (Peterson et al. 2003). A meta-model approach could enhance the creation of plausible alternative management scenarios, and generate deeper insight into the consequences of different actions on the wildlife species or population under analysis, by providing opportunities for participants to develop scenarios for each submodel. 
Fig. 1. Sample outputs from VORTEX (left), OUTBREAK (right), and SPATIAL (bottom) programs.

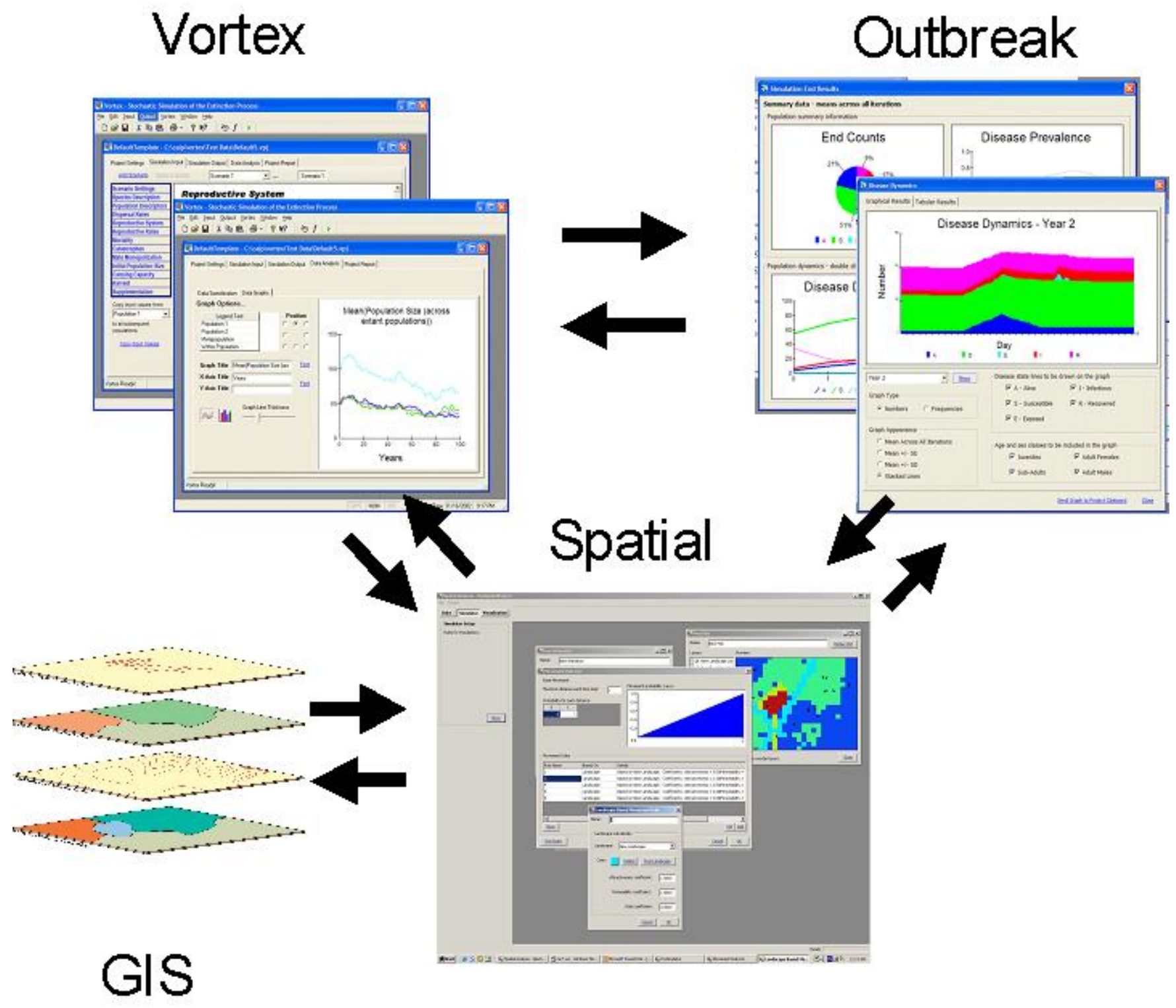




\section{IS THERE A ROLE FOR META-MODELS AND BIOCOMPLEXITY IN BIODIVERSITY CONSERVATION?}

As Munns (2006) points out, there is a continuing need to develop research strategies to understand the impact and interaction of multiple stressors for conservation and policy formulation. Our metamodel and multi-stakeholder approach (see also Gray 1989, Westley and Vredenburg 1997, Phillips et al. 2000, Lewicki et al. 2002, Bertels and Vredenburg 2004) provides one framework for tackling the complexity inherent in broadening biodiversity risk assessment analysis beyond simply genetics and wildlife populations to incorporate inputs from the physical and human domains.

Will biocomplexity help to solve the extinction crisis? Realistically, not in the near term. But "good conservation requires good science" (Tilson et al. 2000). Many of the most effective conservation approaches we use today, from ecosystem-based conservation to adaptive management, to hot spots, etc., came out of innovative and crosscutting research. Aldo Leopold, the founder of modern wildlife management, came to the realization threequarters of a century ago that systems are the critical unit of management concern (Leopold 1970). Many more resources are required to fight the extinction crisis in the villages of Africa, the government offices of Asia, the sprawling urban developments of the Rocky Mountain west, and the executive suites of resource extraction companies developing Amazonian South America. But if history is any indication, research to better understand the linkages among coupled human-natural systems and the biodiversity crisis today will lead to more effective conservation and environmental policy action tomorrow. In the end this comes down to a fundamental philosophical question about the role of research in solving our future environmental problems. Simple research or field conservation efforts alone are unlikely to solve the crisis. But a clear understanding of complex system function, including those social processes involved in fostering this intellectual clarity, is an important catalyst for those individuals and institutions on the ground fighting to protect our world's remaining biological heritage.
Responses to this article can be read online at: http://www.ecologyandsociety.org/voll2/iss 1/art31/responses/

\section{Acknowledgments:}

This material is based on work supported by the National Science Foundation under Grant Nos. 0083615 and 0296181. We acknowledge the contributions of other members of the network, including Onnie Byers, George Francis, Gayl Ness, and John Williams. We particularly acknowledge the inspiration of Ulysses S. Seal.

\section{LITERATURE CITED}

Beissinger, S. R., and D. R. McCullough. 2002. Population viability analysis. Chicago University Press, Chicago, Illinois, USA.

Bertels, S., and H. Vredenburg. 2004. Broadening the notion of governance from the organization to the domain. in I. Demirag, editor. Towards Better Regulation, Governance and Accountability: Global Perspectives from Corporations and Civil Society. Greenleaf Publishing, Sheffield, U.K.

Boyce, M. S. 1992. Population viability analysis. Annual Review of Ecology and Systematics 12:481-506.

Brook, B. W., J. J. O'Grady, A. P. Chapman, M. A. Burgman, H. R. Akçakaya, and R. Frankham. 2000. Predictive accuracy of population viability analysis in conservation biology. Nature 404:385387.

Brown, M. T., and S. Ulgiati. 2004. Energy quality, emergy, and transformity: $H$. T. Odum's contributions to quantifying and understanding systems. Ecological Modelling 178:201-213.

Burgman, M., S. Ferson, and H. R. Akçakaya. 1993. Risk assessment in conservation biology. Chapman and Hall, New York.

Caughley, G. 1994. Directions in conservation biology. Journal of Animal Ecology 63:215-244.

Clark, T. W., and R. L. Wallace. 1998. Understanding the human factor in endangered 
species recovery: an introduction to human social processes. Endangered Species Update 15:2-9.

Colwell, R. 2001. Biocomplexity. Pages 1-8 in Encyclopedia of life sciences. Macmillan, Basingstoke, Hants, UK.

Ellner, S. P., J. Fieberg, D. Ludwig, and C. Wilcox. 2002. Precision of population viability analysis. Conservation Biology 16:258-261.

Folke, C., S. Carpenter, T. Elmqvist, L. Gunderson, C. S. Hollings, and B. Walker. 2002. Resilience and sustainable development: building adaptive capacity in a world of transformations. Ambio 31:437-440.

Gitay, H., A. Suarez, D. J. Dokken, and R. T. Watson. 2002. Climate change and biodiversity. Intergovernmental Panel on Climate Change, New York, New York, USA.

Gray, B. 1989. Collaborating: finding common ground for multiparty problems. Jossey-Bass, San Francisco, California, USA.

Gunderson, L. H., and C. S. Holling. 2002. Panarchy: understanding transformations in human and natural systems. Island Press, Washington, D. C., USA.

Hall, J., and H. Vredenburg. 2003. The challenges of innovating for sustainable development. MIT Sloan Management Review 45:61-68

Haydon, D. T., M. K. Laurenson, and C. SilleroZubiri. 2002. Integrating epidemiology into population viability analysis: managing the risk posed by rabies and canine distemper to the Ethiopian wolf. Conservation Biology 16:1372-1385.

Heberlein, T. A. 1988. Improving interdisciplinary research: integrating the social and natural sciences. Society and Natural Resources 1:5-16.

Holling, C. S., and L. Gunderson. 2002. Resilience and adaptive cycles. Pages 25-62 in C. S. Holling, editor. Panarchy. Island Press, Washington, D.C., USA.

Holling, C. S., L. Gunderson, and G. Peterson. 2002. Sustainability and panarchies. Pages 63-102 in C. S. Holling, editor. Panarchy. Island Press, Washington, D.C., USA.
Kates, R. W., W. C. Clark, R. Corell, J. M. Hall, C. C. Jaeger, I. Lowe, J. J. McCarthy, H. J. Schellnhuber, B. Bolin, and N. M. Dickson. 2001. Environment and development: sustainability science. Science 292:641-642.

Lacy, R. C. 1993. VORTEX: a computer simulation model for population viability analysis. Wildlife Research 20:45-65.

Lacy, R. C. 2000. Structure of the VORTEX simulation model for population viability analysis. Ecological Bulletins 48:191-203.

Lacy, R. C., and D. B. Lindenmayer. 1995. A simulation study of the impacts of population subdivision on the mountain brushtail possum, Trichosurus caninus Ogilby (Phalangeridae: Marsupialia), in south-eastern Australia. II. Loss of genetic variation within and between subpopulations. Biological Conservation 73:131-142.

Lacy, R. C., and P. S. Miller. 2002. Incorporating human populations and activities into population viability analysis. Pages 490-510 in S. R. Beissinger and D. R. McCullough, editors. Population viability analysis. University of Chicago Press, Chicago, Illinois, USA.

Lamberson, R. H., R. McKelvey, B. R. Noon, and C. Voss. 1992. A dynamic analysis of Northern Spotted Owl viability in a fragmented forest landscape. Conservation Biology 6:505-512.

Leopold, A. 1970. A Sand County almanac. Ballantine Books, New York, New York, USA.

Levin, S. 1999. Fragile dominion: complexity and the commons. Perseus, Cambridge, UK.

Levin, S. A. 2000. Multiple scales and the maintenance of biodiversity. Ecosystems 3:498-506.

Lewicki, R., B. Gray, and M. Elliott, editors. 2002. Making sense of intractable environmental disputes. Island Press, Washington, D.C., USA.

Lindenmayer, D. B., T. W. Clark, R. C. Lacy, and V. C. Thomas. 1993. Population viability analysis as a tool in wildlife conservation policy: with reference to Australia. Environmental Managemen$t$ 17:745-758.

Lindenmayer, D. B., and R. C. Lacy. 2002. Small 
mammals, habitat patches and PVA models: a field test of model predictive ability. Biological Conservation 103:247-265.

Lindenmayer, D. B., R. C. Lacy, and M. L. Pope. 2000. Testing a simulation model for population viability analysis. Ecological Applications 20:580-597.

Lindenmayer, D. B., R. C. Lacy, H. TyndaleBiscoe, A. C. Taylor, K. L. Viggers, and M. L. Pope. 1999. Integrating demographic and genetic studies of the Greater Glider Petauroides volans in fragmented forests: predicting movement patterns and rates for future testing. Pacific Conservation Biology 5:2-8.

Lindenmayer, D. B., and H. P. Possingham. 1995. The conservation of arboreal marsupials in the montane ash forests of the central highlands of Victoria, south-eastern Australia: VII. Modelling the persistence of Leadbeater's possum in response to modified timber harvesting practices. Biological Conservation 73:239-257.

Lindenmayer, D. B., and H. P. Possingham. 1996. Ranking conservation and timber management options for Leadbeater's Possum in southeastern Australia using population viability analysis. Conservation Biology 10:235-251.

Lindenmayer, D. B., H. P. Possingham, R. C. Lacy, M. A. McCarthy, and M. L. Pope. 2003. How accurate are population models? Lessons from landscape-scale tests in a fragmented system. Ecology Letters 6:41-47.

Liu, J. L., J. B. Dunning, and H. R. Pullman. 1995. Potential effects of a forest management plan on Bachman's Sparrows (Aimophila aestivalis): linking a spatially explicit model with GIS. Conservation Biology 9:62-75.

Machlis, G. E. 1998. New forestry, neopolitics, and voodoo economies: research needs for biodiversity management. Pages 5-16 in D. Field, editor. Ecosystem management: adaptive strategies for natural resources organizations in the twenty-first century. Taylor and Francis, Philadelphia, Pennsylvania, USA.

Michener, W. K., T. J. Baerwald, P. Firth, M. A. Palmer, J. L. Rosenberger, E. A. Sandlin, and H. Zimmerman. 2001. Defining and unraveling biocomplexity. BioScience 51:1018-1023.
Miller, P. S., and R. C. Lacy. 2003. Integrating the human dimension into endangered species risk assessment. Pages 41-63 in F. R. Westley and P. S. Miller, editors. Experiments in consilience: integrating social and scientific responses to save endangered species. Island Press, Washington, D. C., USA.

Morris, W. F., P. L. Bloch, B. R. Hudgens, L. C. Moyle, and J. R. Stinchcombe. 2002. Population viability analysis in endangered species recovery plans: Past use and future improvements. Ecological Applications 12:708-712.

Munns, W. R., Jr. 2006. Assessing risks to wildlife populations from multiple stressors: overview of the problem and research needs. Ecology and Society 11(1):23. Online URL: http://www.ecologyandsociety. org/vol11/iss1/art23/.

Nicolson, C., A. M. Starfield, G. P. Kofinas, and J. A. Kruse. 2002. Ten heuristics for interdisciplinary modeling projects. Ecosystems 5:376-384.

Nyhus, P. J., F. R. Westley, R. C. Lacy, and P. S. Miller. 2002. A role for natural resource social science in biodiversity risk assessment. Society and Natural Resources 15:923-932.

Odum, H. T. 1996. Environmental accounting: emergy and environmental decision making. Wiley, New York, New York, USA.

Odum, H. T., and E. C. Odum. 2000. A prosperous way down: principles and policies. Colorado University Press, Boulder, Colorado, USA.

Peterson, G. D., G. S. Cumming, and S. R. Carpenter. 2003. Scenario planning: a tool for conservation in an uncertain world. Conservation Biology 17:358-366.

Phillips, N., T. B. Lawrence, and C. Hardy. 2000. Interorganizational collaboration and the dynamics of institutional fields. Journal of Management Studies 37:23-43.

Pickett, S. T. A., W. R. Burch Jr, and J. M. Grove. 1999. Interdisciplinary research: maintaining the constructive impulse in a culture of criticism. Ecosystems 2:302-307.

Plentovich, S., J. W. Tucker, N. R. Hollern, and 
G. E. Hill. 1998. Enhancing Bachman's sparrow habitat via management of red-cockaded woodpeckers. The Journal of Wildlife Management 62:347-354.

Redman, C. L. 1999. Human dimensions of ecosystem studies. Ecosystems 2:296-298.

Reed, J. M., L. S. Mills, J. B. Dunning Jr., E. S. Menges, K. S. McKelvey, R. Frye, S. R. Beissinger, M.-C. Anstett, and P. S. Miller. 2002. Emerging issues in population viability analysis. Conservation Biology 16:7-19.

Sjögren-Gulve, P., and T. Ebenhard, editors. 2000. The use of population viability analyses in conservation planning. Munksgaard International, Copenhagen, Denmark.

Somerville, M., and D. Rapport, editors. 2000. Transdisciplinarity: re-creating integrated knowledge. UNESCO/EOLSS, Oxford, UK.

Schumaker, N. T., T. Ernst, D. White, J. Baker, and P. Haggerty. 2004. Protecting wildlife response to alternative future landscapes in Oregon's Willamette Basin. Ecological Applications 14:381-400.

Swart, R., P. Raskin, J. Robinson, R. Kates, and W. C. Clark. 2002. Critical challenges for sustainability science. Science 297:1994-1995.

Tilson, R., P. Nyhus, P. Jackson, H. Quigley, M. Hornocker, J. Ginsberg, D. Phemister, N. Sherman, and J. Seidensticker. 2000. Securing a future for the world's wild tigers. Save The Tiger Fund, National Fish and Wildlife Foundation, Washington, D.C., USA.

Vanclay, J. K. 1998. FLORES: for exploring land use options in forested landscapes. Agroforestry Forum 9:47-52.

Vinebrooke, R. D., K. L. Cottingham, J. Norberg, M. Scheffer, S. I. Dodson, S. C. Maberly, and U. Sommer. 2004. Impacts of multiple stressors on biodiversity and ecosystem functioning: the role of species co-tolerance. Oikos 104: 451-457.

Vredenburg, H. 2003. Strangers at the party. Pages 298-322 in F. R. Westley and P. S. Miller, editors. Experiments in consilience: integrating social and scientific responses to save endangered species.
Island Press, Washington, D.C., USA.

Watson, R. T. 2001. Climate change 2001: synthesis report third assessment report of the intergovernmental panel on climate change. Cambridge University Press, Cambridge, UK.

Wear, D. N. 1999. Challenges to interdisciplinary discourse. Ecosystems 2:299-301.

Westley, F. R., and P. S. Miller, editors. 2003. Experiments in consilience: integrating social and scientific responses to save endangered species. Island Press, Washington, D.C., USA.

Westley, F. R., and H. Vredenburg. 1997. Interorganizational collaboration and the preservation of global biodiversity. Organizational Science 8:381-403.

Westley, F. R., P. S. Miller, and R. C. Lacy. 2003. Far from land: further explorations in consilience. Pages 352-361 in F. R. Westley and P. S. Miller, editors. Experiments in consilience: integrating social and scientific responses to save endangered species. Island Press, Washington, D.C., USA.

Westley, F. R., and H. Vredenburg. 2003. The art of walking through walls: strategy and structure in the Conservation Breeding Specialist Group. Pages 23-40 in F. R. Westley and P. S. Miller, editors. Experiments in consilience: integrating social and scientific responses to save endangered species. Island Press, Washington, D.C., USA.

Wilson, E. O. 1998. Consilience: the unity of knowledge. Knopf, New York, New York, USA.

Wondolleck, J., and S. Yaffee. 2000. Making collaboration work. Island Press, Washington, D. C., USA. 